\title{
A PROPOS DES LAITS DE MONTAGNE
}

\author{
par
}

\author{
Docteur J. VIEILLY \\ Vétérinaire.
}

Une des questions intéressantes en Industrie laitière, jusqu'alors délaissée, est celle qui concerne particulièrement les laits de montagne. Le XII ${ }^{e}$ Congrès international, qui devait tenir ses assises en 1940, proposait l'étude du climat d'altitude et l'influence de ses différents facteurs sur les fourrages, les bêtes laitières, le lait et les produits dérivés du lait. Nous voulons, sur ce sujet de la laiterie en montagne, faire part de quelques réflexions.

Il nous paraît d'abord illogique de proposer l'étude du seul lait des montagnes des Alpes, considérées comme le prototype des montagnes terrestres. On ne peut, cependant, établir de parallèles comparables entre les laits obtenus dans les montagnes d'Espagne, d'Angleterre, de Russie, de Norvège, etc., pour ne citer que l'Europe, et "a fortiori " dans les montagnes des autres continents, sauf peut-être certaines régions de la Nouvelle-Zélande, du Canada ou du Caucase, par exemple, permettant d'acclimater des espèces animales analogues aux nôtres.

La vache, la brebis, la chèvre sont des animaux d'importation relativement récente dans nos massifs montagneux et je crois que pour bien comprendre le comportement de ces animaux en altitude, il faut ramener leur mode de vie à celui du roi de la montagne : le Chamois, qu'il est important de bien connaître.

A ce sujet, nous ne pouvons que conseiller la lecture agréable et pleine d'enseignements d'un livre du $\mathrm{D}^{\mathrm{r}}$ Marcel A. J. Couturier, paru récemment : "Le Chamois ", édité par la Librairie Arthaud de Grenoble.

Le chamois est le plus gros ancien mammifère à avoir subsisté dans nos montagnes. La répartition géographique du chamois, il y a quelques siècles, représente une zone d'altitude devenue la zone d'habitat de la vache, de la brebis, de la chèvre.

Ces animaux vivent maintenant temporairement en alpages, pendant la belle saison, en des endroits où seul, dans le temps, vivait le chamois.

De nos jours encore, le chamois n'hésite pas à descendre très bas des zones où il a été repoussé, en général par l'homme, retrouvant dans les alpages aux environs de 2.000 mètres, la nourriture de ses ainés que consomment actuellement nos mammifères domestiques.

Chez le chamois, roi de la montagne, qui sait choisir sa nourriture, 
le rachitisme est inconnu actuellement. Il peut done nous servir d'exemple et nous montrer dans quelle direction nous devons diriger nos efforts pour la laiterie en montagne et pour l'obtention d'un lait riche et complet.

Je citerai l'analyse d'un lait de chamois tué le 7 octobre, par le Dr Marcel A. J. Couturier, à moins de 2.000 mètres, à Lauvitel (Oisans), dans une région où dans le même temps et à la même altitude se trouvent encore aux pâturages des vaches, des brebis et des chèvres.

Grammes

Taux de matière grasse (méthode d'Adam) ......... 250

Taux des matières protéiques ................ 80

Taux de sucre réducteur. . . . . . . . . . . . 17

Ainsi ces aliments que les chamois ont mangés pendant des millénaires, nos femelles domestiques les mangent à l'état frais pendant la belle saison et à l'état sec pendant l'hivernage, ce fourrage étant récolté dans des pâturages les plus accessibles, en majeure partie composés de prairies naturelles. (Il n'y a pas 10\% de prairies artificielles parmi les hauts alpages les mieux partagés.)

Cependant, pour toutes ces questions fourragères, il importe de ne pas oublier de tenir compte d'un facteur qui joue un très grand rôle partout et surtout en montagne, c'est le facteur soleil.

Le soleil par ses radiations actiniques augmentant considérablement la quantité de vitamine $\mathrm{D}$ dans les fourrages verts et surtout dans les fourrages secs (séchés sur place), et sachant de plus que les sels de chaux sont ionisés par ces mêmes radiations, le métabolisme du calcium se trouve ainsi facilité.

Partout, done, où le soleil aura pu irradier les nourritures solides et liquides de nos mammifères, le rachitisme sera une rareté; partout au contraire où cette irradiation sera faite parcimonieusement, le rachitisme apparaîtra avec toutes ses conséquences secondaires; propension à la tuberculose, gravité de la fièvre aphteuse, lait peu riche en certains éléments entraînant le rachitisme des enfants de l'homme (carie dentaire, etc.).

Tout celà montre qu'il y a en montagne du bon, du très bon lait, mais qu'il y a aussi des laits inférieurs. Il est nécessaire de tenir compte à ce sujet de facteurs auxquels on n'a pas jusqu'à présent porté d'attention suffisante, notamment l'exposition des prairies et les couches géologiques sur lesquelles celles-ci sont établies :

"Prairies à l'avers, à l'adret, au nord, au sud, en sous-bois, en alpages, ensoleillées, caillouteuses, etc. ", pouvant avoir la même flore entre elles (mises à part quelques plantes spécifiques).

Il est clair, ainsi, que la valeur de la production laitière de nos 
mammifères domestiques est fonction de la valeur biologique totale de ces prairies.

Comme je l'ai déjà écrit, les crus laitiers sólectionnśs proviennent de régions spéciales où tous les animaux s'alimentent dans les mêmes conditions, non seulement avec des plantes sṕ́ciales poussant sur un terrain adéquat, mais avec des plantes nourries et séchées en plein soleil.

Cette sélection de la nourriture, faite par le chamois, pouvant donc s'appliquer par extension à nos mammifères domestiques ne peut être mieux indiquée que par ce passage du livre du $D^{r}$ Marcel A. J. Couturier, chapitre IX, page 501, que je veux transcrire ici fidèlement :

"La recherche de la nourriture joue un rôle considérable dans les mours de l'animal, conditionne sa dissémination géographique et l'altitude à laquelle il vit. Le chamois capricieusement trie avec gourmandise ses plantes préférées qu'il sait merveilleusement trouver. On met trop souvent sur le compte de conditions mútéorologiques le choix de certains versants montagneux, en réalité uniquement choisis pour leur provende savoureuse. Je connais ainsi une montagne dont le versant oriental est riche en chamois et recherché des moutons, alors que le versant ouest, à végétation en apparence identique, est complètement délaissé. Bien mieux, les bergers de la commune de ce versant occidental ont toujours beaucoup de peine à empêcher leurs troupeaux de passer l'arête pour aller de l'autre côté brouter l'herbe plus tendre. Les conditions stationnelles en effet modifient d'une façon très appréciable l'aspect et surtout le goût des plantes.

"La végétation alpine, depuis la zone des forêts jusqu'à la région des névés, est riche en plantes de toutes sortes, la plupart parfumées et succulentes, que le chamois sait apprécier. Le fond de sa nourriture est composé de plantes rares, principalement des trèfles, des plantains et de nombreuses graminées. La plante base de son alimentation est le Trifolium alpinum. Ce trèfle à fleurs roses, et dont la forme est très différente de celle des autres trèfles, se trouve dans tous les terrains granitiques des Alpes et des Pyrénées. Dans les Alpes, il est communément appelé "pied de poule", en raison de la disposition de ses trois longues folioles. Dans les Pyrénées et surtout sur le versant espagnol, cette légumineuse, dont l'isard raffole, est connue sous le nom de "réglisse ", ses tiges et surtout ses racines qui sont très développées ont une saveur sucrée qui rappelle parfaitement le goût de la réglisse officinale. Quand des chamois ou des isards ont découvert une grande pelouse de Trifolium alpinum, ils en mangent jusqu'à satiété, sans chercher ailleurs d'autres plantes. Parmi les autres trèfles appréciés par le chamois, il faut 
citer le Trifolium pratense qui de la plaine remonte assez haut dans la montagne, le $T$. alpestre aux gros capitules, le $T$. montanum, le $T$. thymoflorum, le $T$. badium aux fleurs jaunes, le $T$. saxatile, enfin le $T$. thalii ou trèfle gazonnant, rampant au ras du sol. Les plantains plus spécialement recherchés sont le Plantago montana, le Plantago alpina (le premier se rencontre plus haut que le second) et le Plantago serpentina. Ses graminées préférées sont: des paturins, surtout Poa alpina, Poa la:ca, P. caesia ; - un vulpin, l'Alopecurus gerardl ; - des avoines, Avena montana, A. sempervirens, Avena versicolor; plusieurs fétuques, Festuca alpira, F. halleri, F. spadicea; l'Elymus europaeus, fréquent dans les bois du massif de la Chartreuse. En été ce que l'animal apprécie le plus dans les graminées, c'est l'épi avant qu'il soit mûr. S'il affectionne les jeunes pousses de certaines joncacées, comme Luzula spadicea surtout, il dédaigne les grosses touffes rêches du dactyle pelotonné, les herbes hautes et rudes vivant dans les lieux humides, comme les Carex ou les Culamagrostis. Il n'aime pas non plus les plantes à odeur forte comme la menthe, l'origan ou l'armoise; je n'ai jamais observé, dans les rochers escarpés habités par le chamois, les plans épars du génépi raccourcis par ses dents.

"Il est cependant des ombellifères qu'il recherehe telles que le Meum mutellina, l'Imperatoria Ostruthium; des crucifères, comme l'Hutchisia alpina, la lunetière à fruits lisses (Biscutella laevigata) et le Brassica Richerii ; - des Polygonées, comme Oxiria digyla ; des composées comme le Senecio Doronicum, l'Adenostyles leucophylla aux fleurs couleur vieux rose, le rare Doronicum pardalianches, mais surtout le Doronicum grandiflorum (Aronicum scorpicides), si répandu dans les Alpes et appelé par les Allemands "herbe à chamois ». Il recherche avidement cette humble plante aux fleurs jaunes dont les pétales périphériques sont d'une belle couleur orangée, le Crepis pygmaea, si abondant dans les clapiers. Dans la montagne pyrénéenne, l'isard ereuse avec les pattes de véritables cavités pour s'emparer de ces tiges blanches et tendres, protégées par des pierres.

"Il aime se rafraîchir avec les feuilles de rosacées du genre Alchemilla, avec celles du framboisier (Rubus Idaeus), d'une ronce naine à fruits rouges, Rubuss axatilis, très répandue dans les clapiers, des groseilliers sauvages, Ribes alpinum à baies douces, $R$. rubrum, R. petraeum à fruits acides, et aussi avec celles du fraisier.

"Il tond, sans inconvénient pour sa santé, les parties florales de Digitalis grandiflora et d'Aconitum lycoctonum (ou aconit tueloup), cette dernière pourtant redoutable pour certains anirnaux. "Notre bête ne vit pas seulement de ces végétaux, mais la nécessité l'oblige bien des fois à se contenter d'une nourriture moins 
choisie dans laquelle entrent la plupart des plantes des montagnes. Le chamois n'aime ni l'herbe mouillée ni l'herbe givrée. Il trouve une agréable diversion à brouter "dente superbo ", debout sur ses pattes de derrière, bourgeons, jeunes pousses et rameaux de certains arbres ou arbustes, comme le cytise, le sorbier nain (Sorbus chamaespilus), l'amelanchier (Amelanchier vulgaris), les vernes, les aulnes (Alnus viridis et Alnus incana), sous lesquels il aime se coucher et trouver la fraîcheur; il est moins tenté par les chèvrefeuilles, le frêne et le noisetier. Il lui plaît aussi de se reposer sur les tapis vert et rouge des rhododendrons et des busseroles (Arbutus uvaursi), mais boude une aussi coriace pâture.

"Il ajoute rarement à son menu des baies et fruits. J'ai vu des groseilliers dont toutes les feuilles avaient été mangées et dont les fruits restaient intacts. Souvent cependant, dans l'estomac de chamois tués en septembre, j'ai trouvé les fruits avalés de l'airelle (Vaccinium myrtillus). Un mâle tué à Valjouffrey (Dauphiné) avait la panse remplie de fruits d'églantier.

"Ainsi que la chèvre domestique, le chamois est friand de certains champignons, en particulier des bolets et des lactaires. Le guidechasseur Joseph Delmas a plusieurs fois tué des isards en train de se repaître de lactaires délicieux (Lactarius deliciosus). A la fin de septembre et en octobre, les isards descendent dans les bois à la recherche des eryptogames. Il est vraisemblable qu'isards et chamois mangent encore d'autres espèces de champignons. ”

\section{LA TENEUR EN MATIËRE GRASSE DU LAIT DE FEMME}

\section{Ses variations au cours de la période de lactation et en fonc- tion de l'âge, de la qualité de bonne nourrice, des condi- tions du début de la menstruation et des circonstances de l'accouchement (1).}

\section{par}

Helmut GÖLZ, Assistenz-Arzt

de Stu.tgart

Après la naissance, les conditions de nutrition deviennent, pour l'enfant, entièrement nouvelles. En contact étroit jusqu'alors avec la mère, il joue maintenant un rôle indépendant, il est devenu un être indépendant. La brusquerie de ce changement se trouve un peu atténuée du fait que l'organisme maternel fournit au nourrisson pendant un certain temps une nourriture standardisée dans ses

(1) Traduction L. ZIVY. 